\title{
Using Structural Computing to Support Information Integration
}

\author{
Kenneth M. Anderson and Susanne A. Sherba \\ Department of Computer Science \\ University of Colorado, Boulder \\ $430 \mathrm{UCB}$ \\ Boulder CO 80309-0430 \\ $\{$ kena, sherba\}@cs.colorado.edu \\ WWW:http://www.cs.colorado.edu/users/\{kena, sherba\}/
}

\begin{abstract}
Software engineers face a difficult task in managing the many different types of relationships that exist between the documents of a software development project. We refer to this task as information integration, since establishing a relationship between two documents typically means that some part of the information in each document is semantically related. A key challenge in information integration is providing techniques and tools that manage and evolve these relationships over time. The structural computing domain provides a set of principles to derive new techniques and tools to help with these tasks of relationship management and evolution. We present a prototype information integration environment, InfiniTe, and describe how we are exploiting structural computing principles in the design of its infrastructure services.
\end{abstract}

\section{Introduction}

Software development projects produce a wide variety of software artifacts, including requirements and design documents and source code. A key challenge in software development is managing the relationships that exist between these documents. For instance, software engineers need to know how a particular source code file relates to the modules and subsystems defined in the project's design document, and how these elements relate to the project's requirements. A benefit of having this information is the ability to perform change impact analysis, (e.g. if I change the interface to this subsystem, how does that impact its ability to meet its requirements, and what source code files need to change?) We refer to this relationship management task as information integration, since establishing a relationship between two documents typically means that some part of the information in each document is semantically related.

We are designing and developing an information integration environment, InfiniTe [5] (pronounced "infinity"), to provide tools to software engineers that increase their ability to discover, manage, and evolve the relationships that exist between their software artifacts. Managing and evolving relationships is a particularly important aspect of this work, since the creation and/or discovery of 
relationships in a software development project is typically only the "tip of the iceberg" of the relationship management problem facing software engineers. The initial requirements and design stages of a large, complex software system represents only $25 \%$ of the total lifetime of a software system. That is, large software systems spend most of their time being used, maintained, and evolved, and a key maintenance challenge is keeping a deployed software system consistent with the software artifacts that represent its documentation.

Structural computing is an emerging field of research exploring a new paradigm of computation based on structure as opposed to data. Peter Nürnberg emphasizes this point of view by stating that structural computing "asserts the primacy of structure over data" and that computing should be performed on entities that are intrinsically structured [10]. Stated another way, the "atoms" of structural computing should have the ability to both contain (structured) information and participate in arbitrary structural relationships. We believe that structural computing can provide critical design guidance to the infrastructure of an information integration environment, especially with respect to this notion of a structural "atom."

In the past, we have argued that structural computing can be usefully applied to the domain of software engineering [2]. This paper represents an initial attempt to apply structural computing principals to the software engineering problem of information integration. The rest of this paper is organized as follows. We briefly present the InfiniTe architecture. Then, we discuss the structural computing issues surrounding the design of InfiniTe's infrastructure services. Next, we briefly discuss related work, and then offer a few conclusions.

\section{InfiniTe Information Integration Environment}

Fig. 1 presents a model of the conceptual elements of the information integration environment. The model consists of users, data sources, integrators, translators, contexts, a repository, and an open hypermedia layer. The basic idea is that information is brought into the information integration environment by invoking a translator. The translator retrieves information from a data source and stores it in a repository. The repository consists of multiple contexts; contexts store information (using XML [6]) along with attributes, which serve to provide meta-data on the information. Contexts can contain sub-contexts. In addition, the documents of a context can be linked together in arbitrary relationships; relationships can span multiple contexts and can have type information associated with them (e.g. a requirements traceability link, a consistency relationship, etc.). Relationships can be stored as XLinks [8] or within the open hypermedia layer.

Integrators aid users in finding, creating, maintaining, and evolving relationships within the repository. While our main focus is on relationship management, integrators are free to create new contexts and to store information within them while performing their tasks. For instance, an integrator that searches documents for particular keywords, may store the location of discovered keywords in 


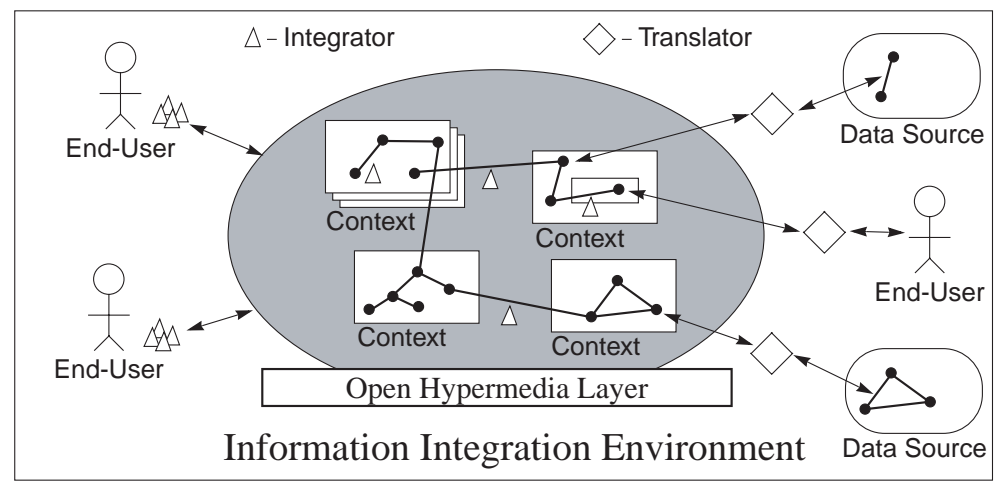

Fig. 1. InfiniTe's Conceptual Model

a document separate from the documents being searched. It may then create a new context and include pointers to the searched documents plus the document that it created.

Finally, the relationships that are generated by integrators can be imported into the open hypermedia layer and then made available to the original data source using its native editing tools. This feature allows software engineers to submit a set of artifacts to the repository, make use of integrators to discover relationships contained in those artifacts, and then view the generated relationships within the native editing environment of the original artifacts. This capability is critical to enabling the adoption of the information integration environment, and is discussed in more detail below; while it is true that our approach requires the use of a new environment to perform information integration tasks, the results of those operations can be made available to the software engineers within their own tools. Software engineers, thus, gain access to tools and techniques to perform information integration tasks, but they are not required to give up the tools they already use to gain these capabilities.

We have implemented a proof-of-concept prototype of InfiniTe to explore a variety of research issues (See Figure 2). The implementation makes use of a set of Java Servlets that can invoke integrators and translators on XML documents stored in the repository. XSLT [7], the transformation component of the XML Stylesheet Language, is used to translate repository information from XML into HTML for presentation in a Web browser. In addition, repository relationships can be extracted from the environment as a set of XLinks that can then be imported into an open hypermedia system for display in the documents of integrated third-party applications. 


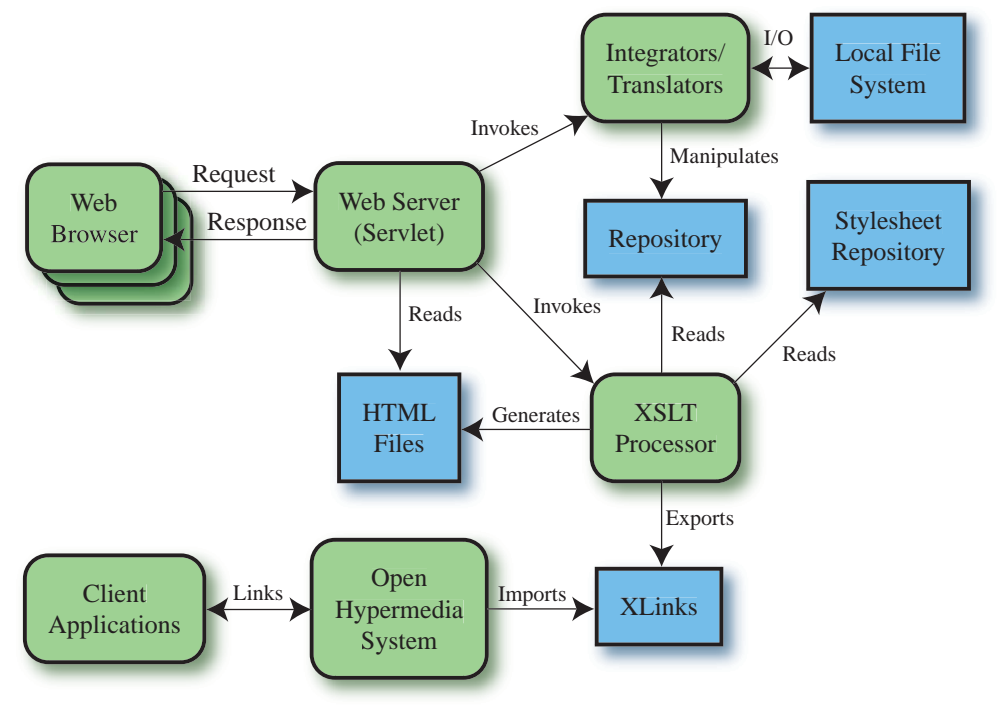

Fig. 2. InfiniTe's Prototype Architecture

\section{$3 \quad$ Structural Computing Issues}

Our use of XML as a means for storing documents and relationships in InfiniTe's repository enabled the rapid creation of a prototype system to explore information integration research issues directly. However, it is currently not clear that XML will prove to be the right choice with respect to its ability to scale to modern software development projects, or its ability to flexibly specify heterogeneous types of relationships.

As we have reported in previous work [1], modern software development projects can quickly test the scalability of software tools. In that particular project, our open hypermedia system, Chimera [4], had to support the creation of hyperwebs containing hundreds of thousands of relationships, and indicated that true industrial support would require hypermedia technology that can scale to hyperwebs containing millions of relationships. With respect to the problem of defining heterogeneous sets of relationships, XLink [8] is certainly a step in the right direction. XLink provides the ability to specify relationships external from the documents they relate and has the ability to assign types to links to help distinguish between different XLink instances. However, XLink is a relatively new standard with sparse tool support and its typing mechanisms relies on XML Namespaces, which is a somewhat difficult-to-use standard that has generated significant controversy in the XML community [9].

As such, we are designing an implementation of the repository for InfiniTe that is based upon structural computing principals. We believe the use of structure servers [10] and a carefully-designed set of structural abstractions can ad- 
dress both of the potential problems raised above, and provide a flexible platform for adding new information integration capabilities and strengthening our integration with open hypermedia technology. We now describe our design of the structural computing-based repository and our plans for implementing and evaluating it.

\subsection{Structural Abstractions}

Our design for InfiniTe's repository, using structural computing principals, is centered around the concept of a file. In InfiniTe, a file will be the structural atom, upon which all other structures are based. As defined by Nürnberg, a file will have the ability to contain structured information as well as participate in structural relationships. The repository will be accessed via two structure servers. One server will provide access to structural abstractions within a file (known as the repository file server), while the other server will provide access to structural abstractions between files (known as the repository structure server). These servers will be hosted on top of a database (most likely a relational database, although XML databases will be evaluated) to help address our scalability concerns.

The repository file server is used to create and access files within the repository. Translators will be the primary user of the repository file server, either storing new files into the repository or extracting files from the repository to a (local or remote) file system. Integrators will also access this server when they are examining the contents of a file looking for relationship instances. This server will provide operations to create files and define their internal structure. Internal structure in the current implementation is provided by the structural aspects of XML. The current repository does not impose a canonical XML format to which all files must conform. Instead, it allows any XML document to be stored in the repository. The tradeoff associated with this design choice is that some translators/integrators may not be able to interact with a particular file if it does not understand its XML structure. The use of a structure server should help to alleviate this problem since a structure server will provide reflection methods that will enable a translator or integrator to query and search the structure of a file dynamically, trying to discover substructures that it can understand and manipulate. Methods for defining the structure of a file will initially support the creation of tree structures but will eventually support matrix and directed graph structures as well. The final set of methods provided by the repository file server concern the creation and manipulation of anchors. Anchors are created by integrators when they discover a structure in a file that they can classify. These anchors can then be used by the repository structure server to establish relationships between files. Note that anchors can be assigned types and queries can be made against those types, e.g. retrieve all anchors of type A from document B.

The repository structure server is used to establish and access the relationships between files in the repository. The primary users of this server will be integrators, who need these services in order to perform their primary duty of 
finding and manipulating InfiniTe relationships. The repository structure server treats files as atoms, not caring about their internal structure. Instead, files can be grouped in contexts and linked with relationships. Operations will be provided to create, edit, query, link, and destroy contexts. Contexts can have relationships established between them: For instance versioning relationships, that express that one context is an earlier or subsequent version of some other context. Operations will also be provided to create, type, edit, query, and destroy relationships. Relationships require anchor points to link files with each other (although both a file and context can serve as its own anchor point). In addition, relationships can be typed, which can constrain both the number and type of anchors that can be related by a particular relationship. With these operations, integrators can be given a starting context or file as input, and use the repository structure server to access other files, either by querying the context, or retrieving a set of relationships from the input file and traversing them to reach related files.

\subsection{Structural Transformation}

In response to our previous work on structural transformations [3], we have identified the need for structural transformation services in the InfiniTe environment. In particular, in software development projects, relationships between software artifacts may change in both structure and purpose over time. For instance, a subsystem may always maintain a relationship between itself and the most recent version of a particular module. As that module is changed, new versions are created, and the subsystem relationship must be modified to point to the new version automatically. Another example involves the changing of a relationship's type. For instance, a relationship that is used to perform change impact analysis in a maintenance context, may need to serve the role of a requirements traceability link in a software understanding context. As such, the same instance of a relationship needs to be viewed as different types depending on the context from which it is accessed.

In order to support these scenarios, our structure servers will provide operations for defining temporary sets of relationships, known as change sets, to which type transformations can be applied. This will support scenarios such as retrieve all change impact relationships from context A and reify them as requirements traceability relationships in context B. In addition, operations will be provided to associate computations with relationships to handle scenarios like the versioning scenario described above. Essentially this capability requires that behaviors that return anchors can be treated as anchors themselves. When such an anchor is accessed, the computation is triggered which retrieves and returns the anchor which is to be used in that instance. This technique is, of course, borrowed from the open hypermedia domain [11] where many systems provide this dynamic linking capability. 


\subsection{Implementation and Evaluation Plans}

The work described in this paper is at a preliminary stage. The initial XML-based prototype of the InfiniTe environment has just been completed. This prototype allows us to explore interesting information integration issues now, while the design and implementation of the structural computing-based repository takes place. We intend to integrate the two identified structure servers incrementally, starting with basic support to store and retrieve files and then adding services such as organizing files into contexts, adding anchors to files and establishing relationships between anchors.

We intend to evaluate our work by applying the information integration environment to several open source projects. Open source projects provide a wealth of software artifacts to analyze, including all artifacts of all previous versions of an open source system. Such projects will allow us to test InfiniTe's scalability as well as its ability to track and transform complex relationships between a heterogenous set of software artifacts.

\section{Related Work}

We now briefly review two related systems. The GeoWorlds environment [13] is an information analysis environment that allows users to retrieve documents off the Web, organize them into collections, and apply a variety of analyses upon them. GeoWorlds is strictly focused on the World Wide Web and can only import information from Web-based data sources. We intend to support both remote and local information sources, with particular attention to supporting legacy, third-party, data formats. This will allow our environment to be applied to both existing and new software development projects. In addition, GeoWorlds services are focused more on information analysis while our focus will be on relationship management. Our environment will thus have greater capabilities for discovering, viewing, and manipulating relationships than what is found in the GeoWorlds environment.

The second related system is xlinkit [12]. It is a link generation engine that allows consistency relationships to be specified over software artifacts. The basic idea is that a software engineer writes consistency rules for a set of documents and then submits those rules along with a set of documents. (Documents must be converted to XML before the link generation engine can process them.) The link generation engine then checks the documents to see if they follow the submitted consistency rules. As output, the engine generates a report that displays the results of the analysis: instances of the rules are highlighted and information is provided to show, for each instance, if the rule was followed or violated. Our environment can be used to check consistency relationships over software artifacts, but it is also intended to support a broader spectrum of relationship types. For instance, we intend to build integrators that can aid the process of generating requirements traceability links, similar to the results we achieved with Northrop Grumman using only the Chimera open hypermedia system [1]. Rather than providing a rule-based language for a single relationship type, our environment 
will provide APIs to software engineers that will allow them to construct their own translators and integrators to manage the relationships relevant to their software development projects. This does not mean that rule-based languages are not helpful in automatic link generation; indeed the experience with xlinkit demonstrates the benefits of this technique. In fact, we plan to leverage the results of the xlinkit experience, along with other work in hypermedia link generation, to create a generic rule-based integrator that can support various rule sets via a plug-in mechanism. In addition, our use of open hypermedia will allow the relationships discovered in the environment to be viewable within the native editing context of the original software artifacts. Thus, while both of these systems require a translation step into XML, our approach will allow information to flow back to the original artifacts.

\section{Summary}

We believe that structural computing principals can be applied to the design of infrastructure services for an environment designed to help software engineers with the problem of information integration. We outlined a design for two structure servers to serve as the repository for the InfiniTe information integration environment centered around a file as the key structural abstraction. We have described the need for structural transformation services in this environment and have provided insight into our implementation and evaluation plans. Although this work is still at a preliminary stage, we believe the design presented in this paper provides utility to structural computing developers as an example of the type of applications that can be addressed by the domain of structural computing.

\section{References}

1. Anderson, K. M. (1999). Issues of Data Scalability in Open Hypermedia Systems. The New Review of Hypermedia and Multimedia, 5: 151-178.

2. Anderson, K. M. (1999). Software Engineering Requirements for Structural Computing. In Proceedings of the First International Workshop on Structural Computing. Darmstadt, Germany. February 21, 1999. <http://www.cs.colorado.edu/users/kena/papers/workshops/sc1.html>.

3. Anderson, K. M. (2000). Structural Computing Requirements for the Transformation of Structures and Behaviors. In Proceedings of the Second International Workshop on Structural Computing. San Antonio, TX, USA.

4. Anderson, K. M., Taylor, R. N., and Whitehead, E. J., Jr. (2000). Chimera: Hypermedia for Heterogeneous Software Development Environments. ACM Transactions on Information Systems, 18(3): 211-245.

5. Anderson, K. M., and Sherba, S. A. (2001). Using XML to support Information Integration. In Proceedings of the 2001 International Workshop on XML Technologies and Software Engineering (XSE 2001). Co-located with the 2001 International Conference on Software Engineering. Toronto, Ontario, Canada. May 15, 2001. 
6. Bray, T., Paoli, J., and Sperberg-McQueen, C. M. (1998). Extensible Markup Language (XML) 1.0, W3C Recommendation, 10-February-1998. $<$ http://www.w3.org/TR/REC-xml>.

7. Clark, J. (1999). XSL Transformations (XSLT) Version 1.0 W3C Recommendation, 16 November 1999. <http://www.w3.org/TR/xslt.html>.

8. DeRose, S., Orchard, D., and Trafford, B. (1999). XML Linking Language (XLINK). <http://www.w3.org/TR/xlink/>.

9. Dumbill, E. (1999). Eight Greats of XML.com 1999. <http://www.xml.com/pub/a/1999/12/bestof/index.html>.

10. Nürnberg, P. J., Leggett, J. J., and Schneider, E. R. (1997). As We Should Have Thought. In Proceedings of the Eighth ACM Conference on Hypertext, pp. 96-101. Southampton, UK. April 6-11, 1997.

11. Østerbye, K., and Wiil, U. K. (1996). The Flag Taxonomy of Open Hypermedia Systems. In Proceedings of the Seventh ACM Conference on Hypertext, pp. 129-139. Washington DC, USA. March 16-20, 1996.

12. <http:// www.xlinkit.com/>.

13. Yao, K., Ko, I., Eleish, R., and Neches, R. (2000). Asynchronous Information Space Analysis Architecture Using Content and Structure-Based Service Brokering. In Proceedings of the 2000 ACM Conference on Digital Libraries. San Antonio, TX, USA. 